\title{
SAFETY RISK ASSESSMENT IN CONSTRUCTION PROJECTS USING INFORMATION MODELS
}

\author{
Vasiliki Manioti ${ }^{1}$, Athanasios Chassiakos ${ }^{2}$, and Stylianos Karatzas ${ }^{3}$
}

\begin{abstract}
The identification of safety risks in construction sites has been a major concern of project managers who need to develop proactive safety plans to reduce accident and health related risks. This paper proposes a method which combines BIM technology, virtual reality tools, and Monte Carlo simulation to reveal possible accident conditions and to assess the probability of accident occurrence with the aim to promote safety management and accident reduction. Within the context of construction projects, the construction site is dynamically modelled employing BIM software. A programming language (Visual $\mathrm{C} \mathrm{\# )}$ is used to represent the human/machinery movements in the virtual environment. Finally, Monte Carlo simulation is performed to analyse specific work scenarios which may lead to hazardous conditions and assess the safety level. The aim is to link the risk output with the BIM model and to develop a heat map of accident risk throughout the project allowing, thus, prompt and effective implementation of preventive measures for accident reduction or avoidance.
\end{abstract}

Keywords: Safety, risk assessment, BIM, virtual reality, simulation

\section{INTRODUCTION}

Safety assessment and management are essential parts of the construction planning process. Despite rigorous efforts of safety professionals and strong governmental reinforcement of safety laws, there has been only limited decay in the frequency and severity of injuries and illnesses in the construction industry (Carter and Smith 2006). Research concerning occupational deaths in construction concludes that the major safety problems are associated to falls, electrocution, struck-by, and caught in/between objects (Goetsch 2012).

Traditional safety planning relies on manual observations, gut-feelings, and experience of the safety planner. Inefficiencies are witnessed in the current methods which are utilized for processing and reporting safety and health related issues in construction projects (Abraham et al. 2004). A typical safety plan depicts what safety measures are necessary, when, where, and why. The link between safety planning and work execution is often weak. For example, many contractors use two-dimensional drawings (2D) or field observations to identify hazards. Since their approach is manual and based on experience, the observed results can be error-prone due to subjective judgment of the decision maker (Sulankivi et al. 2012).

1 Graduate Student, Department of Civil Engineering, University of Patras, Patras, Greece, vasiliki.manioti@gmail.com

2 Associate Professor, Department of Civil Engineering, University of Patras, Patras, Greece, a.chassiakos@upatras.gr

3 PhD Candidate, Department of Civil Engineering, University of Patras, Patras, Greece, ksteliosk@hotmail.com 
Many efforts for safety analysis and control have been based on historical safety statistics. For example, Saurin et al. (2004) integrated a Safety Planning and Control Model (SPC) into the production planning and control process. Rozenfeld et al. (2010) developed a specific Construction Job Safety Analysis (CJSA) tool. Tam et al. (2002) applied Non-Structural Fuzzy Decision Support System (NSFDSS) to evaluate safety management systems and prioritize measures with the consideration of various decision criteria to facilitate more realistic decision making. These are, however, not sufficient to predict when and where accidents occur in every single construction project. For safety management, the detection of possible accident-prone conditions and the assessment of the likelihood and impact of potential accidents are required. Given the dynamic characteristics of a construction project and the temporary nature of working conditions, accident risks also tend to have a dynamic form.

This has led to the advent of information technology enabled approaches for construction safety using virtual designs and simulations of construction operations. Technology that assists construction safety experts in the task of easier recognizing and resolving safety hazards while addressing the complexity and dynamism of jobsite conditions can lead to safer construction with less effort (Ku and Mills, 2010). Recently, there has been considerable interest in improving worksite safety though safer design and work method statements using Building Information Modelling (Chi et al. 2012).

It has been realized that BIM can be utilized to promote safety management and combine safety with other construction planning processes. Turner Construction established a standard model-checking procedure to ensure their project compliance with rigorous standards of safety by developing a rule set package on Solibri Model Checker (SMC) (Downey, 2012). The VTT Technical Research Center of Finland has developed a detailed framework for fall protection modelling and 4D visualization (Kiviniemi et al., 2011). Another tool for the automatic construction safety analysis is the application of a safety rule checking algorithm to BIM with the objective to improve safety on construction sites regarding fall-related hazards and based on object-oriented building models (Zhang et al. 2013).

Most existing studies employ deterministic approaches which can provide alerts for specific incidents (isolated in space and time) and following their occurrence (Isaac and Edrei, 2016). The hypothesis of the present research is that a probabilistic type of model can better support a dynamic form of safety management. This is done by providing proactive alerts not only at conditions of clearly high risk but also by complementing the safety evaluation with probabilistic alerts for cases in which the risk may be lower but not negligible. In the proposed approach, BIM technology can be used as a basis for safety planning and communication. The utilisation of BIM can result in improved occupational safety by connecting safety matters to construction planning and execution, providing more illustrative site layout and safety plans, methods for managing and visualising up-to-date plans, and site status information (Sulankivi et al. 2009). In this paper, the BIM digital representation of the physical and operational characteristics of the construction site is combined with Monte Carlo simulation to provide an improved safety risk assessment and decision making tool. This is further supported by developing visualized movements of workers/machinery in a Visual C\# software environment. Results of such development are presented for an indicative case of hazardous conditions in a building construction environment. 


\section{SYSTEM STRUCTURE}

\subsection{Method Description}

Risk assessment for the various project components and functions in the construction site is required in each project stage. Given the difficulty of continuous spot risk measurement and forecasting, an alternative way of dealing with it is to simulate work functions and the environment in a dynamic virtual model throughout the construction period. In this way, it is possible to assess the safety conditions at any time preventing accident occurrence or reducing the resource (manpower and equipment) exposure to it. Based on these considerations, a project risk assessment method is developed within the framework of safety management which includes risk identification, risk assessment, and risk prevention components (Figure 1).

In the risk identification phase, the proposed method applies BIM software for project and construction modelling which can be used in conjunction with an automated safetyrule checking system (in the context of this research possible hazardous locations have been identified manually). In the second phase (which represents the main focus of this research), Monte Carlo simulation and a programming language (visual $\mathrm{C} \#$ ) are employed for the development of the "virtual reality" scheme and the assessment of the risks identified in the previous phase. In the third phase, numerical risk outputs could be linked to BIM to reflect high risk locations and periods. A basic interpretation (i.e. low, medium, high) could be used to create a BIM heat map of accident risk. With this information, risk could be prioritized and preventively managed. For the purpose of better illustration, the following presentation is aligned with the 'worker fall through an opening' case which is further analysed in section 3.

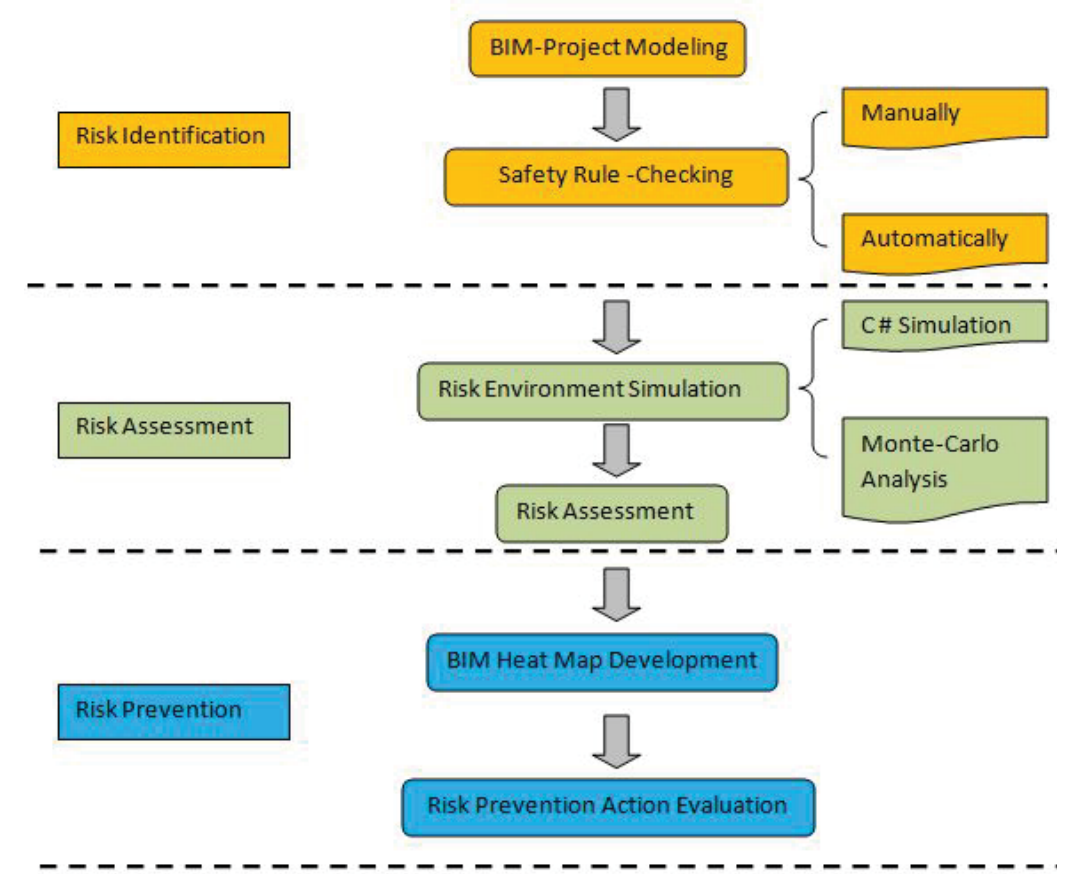

Figure 1: A conceptual model for risk identification, assessment and prevention 


\subsection{Project Modelling}

The project and construction site is initially modelled by BIM software (Revit). This modelling constitutes a unique database that contains the entire information of the structure, including the specific characteristics of each structural component of the project. The first step is the introduction of the general project information. The description of the project, the area, the ground on which it is founded, and its orientation are some essential inputs. The virtual building is developed based on the use of threedimensional parametric objects to construct walls, floors, ceilings, openings, roofs, columns, beams as well as the various electrical systems. Having set a reference level, the creation of various other levels follows in order to achieve proper placement of data and improve the overall design process.

\subsection{Simulation of Worker Movement}

In the present research, the project locations with high risk potential for 'falls through an opening' have been identified manually. Alternatively, existing safety rules, guidelines, and best practices can be used in conjunction with 3D design and schedule information to formulate an automated safety rule checking system. A BIM platform equipped with its own rule engine (i.e. Solibri Model Checker, SMC) can provide users the possibility to self-define or configure safety rules for rule-checking process with the intention to automatically identify hazard locations on the site (Zhang et al. 2015).

For the simulation of a single risk type, the structure and operating conditions at the site should be depicted including the movement of workers/machinery within this environment. Due to the large number and risk types, an indicative case of a 'worker fall through an opening' risk is presented herein at different work scenarios (single worker movement, opposite worker movements, existence of obstacles or protective tools in the worker path). The simulation of worker movement aims to develop a virtual model that clearly depicts the risk characteristics and possible consequences.

The data considered is the site plan created with Revit modelling program. The simulation has been done with the programming language Visual C \# using the Microsoft Visual Studio 2010 code development tool and the .NET Framework. The site plan view is inserted as an image in this program in which the simulation is conducted. In this image, spaces occupied by buildings or temporary installations as well as corridors are portrayed. In the case of 'worker falling through an opening', the movement of the worker on a corridor is illustrated by using pixels representing the worker position. The movement is actually represented by the succession of pixels.

\subsection{Safety Conditions Simulation and Risk Assessment}

The 'fall through an opening' risk is associated with the worker movement trajectory in space and with respect to the opening while the risk depends on several parameters including geometric configuration (location, size, and geometry of openings, length and width of movement corridor, straight-line or curved trajectory), operational characteristics (frequency of movements, obstacles in worker path, load carried, existence of cross movements, slippery conditions, protective measure existence, etc), and the worker tendency, ability, and experience to avoid putting him in danger. The exercise should take into account this very important human factor which is often the most unpredictable (e.g., careless movement, non-compliance with regulations, etc). Due to the "randomness" of human behaviour and actions, simulation needs to include stochastic (probabilistic) data for the risk evaluation. 
The simulation is intended to assess the probability of accident occurrence under given working conditions. The analysis employs Monte Carlo simulation using the Palisade @Risk software with a Microsoft Excel sheet in a number of scenarios and based on parameters concerning:

- the opening length and the corridor width adjacent to the opening,

- uni- or bi-directional worker movement in combination with load carriage which can result in deviations from a 'straight line' trajectory,

- the existence of obstacles which limit the effective width of the corridor or barriers acting protectively.

Each probabilistic parameter takes a random value based on a certain parameter range and a probability distribution. The process is repeated several times and a falling probability distribution is developed. Based on this distribution, aggregate information relating to average probability values can be extracted.

\section{Pilot ApPlicAtion}

The pilot implementation refers to the case that workers are moving from point $\mathrm{C}$ to point $\mathrm{E}$ via point $\mathrm{D}$ (Figure 2). The figure presents a building floor with an opening in the middle surrounded by a corridor leading to the floor rooms. The corridor width is assumed 4.0 meters. Point D is considered as the critical point in terms of risk as the worker trajectory converges towards the opening. The following scenarios are considered for the pilot implementation:

1. An individual worker moves back and forth between points $C$ and E. No protection from falling is available (Figure 2a).

2. More than one workers move along the corridor reducing the effective corridor width when coming across. No protection from falling is available (Figure 2b).

3. An individual worker moves along with an obstacle placed (intentionally or unintentionally) next to the opening reducing the effective corridor width and directing the worker far away from the opening, acting thus as a protective measure (Figure 2c).

The worker trajectory generally coincides with the middle path of the corridor but there are always reasons for deviating from it, e.g. load carriage or debris existence during the construction phase. For this reason, a Pert distribution function is assumed to model the expected deviation of the worker route from the middle way. Further, it is assumed that the risk of falling increases exponentially as the worker approaches the opening (following rough expert judgement). Specifically, the probability falling risk exposure in relation to the cross distance between the worker trajectory and the opening is given by:

$$
P=a\left(\frac{4-d}{4}\right)^{10}
$$

where, $P$ : risk exposure probability, $d$ : distance from opening, $a$ : parameter that indicates the worker capability to avoid falling through the opening (further discussed below). Based on the above equation and with $\alpha=1$, if the worker step on the opening boundary, then $d=0$ and $P=1$ (full exposure to risk). For $d=1$ then $P=0.056$ while for $d>1$, the probability tends to zero. 

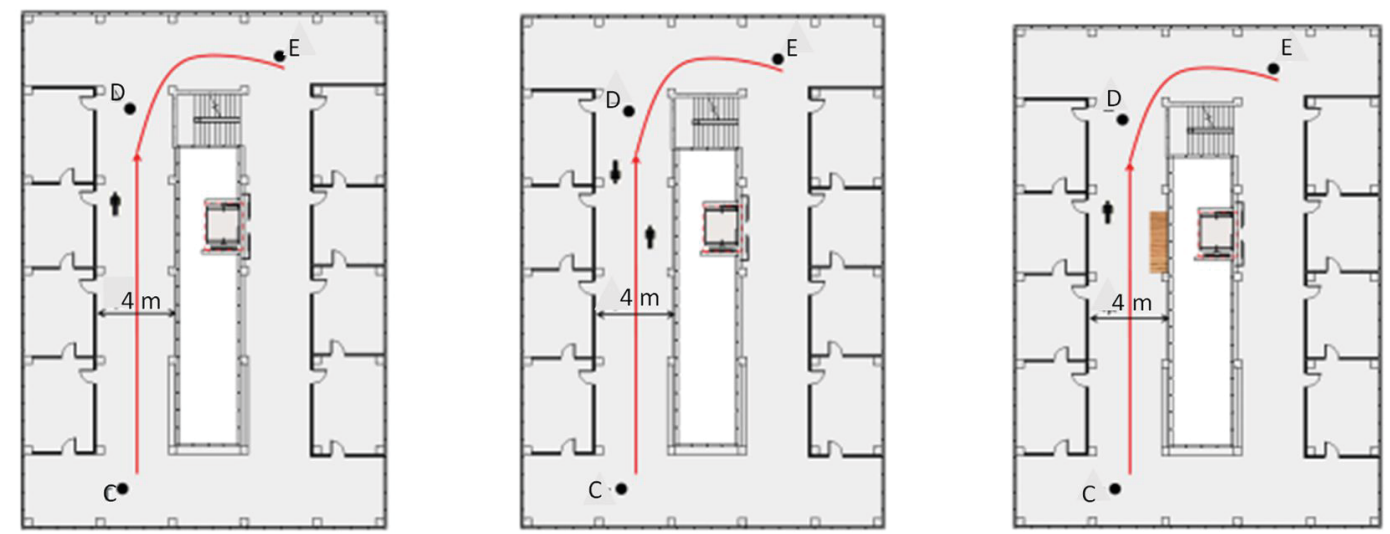

Figure 2: Plan view of a building floor with workers moving around: (a) single worker movement; (b) opposite worker movements; (c) obstacle existence

The final outcome regarding the falling probability depends not only on the worker position in relation to the opening but also on his individual ability to avoid falling. The work experience in similar conditions, the worker self-awareness and responsibility in dealing with risks, the stepping conditions (e.g., slippery floors or floors with debris), the loading conditions (with or without load), and the working conditions (working pressure, anxiety etc.) play an important role in human reaction and risk prevention. For this reason, an additional parameter $\alpha$ is considered in Equation (1) to account for the expected worker ability to respond to risk under certain conditions. The parameter takes values between 30-70\% (indicating that, even if the worker steps on the edge of the corridor adjacent to the opening, the probability to fall is not definitely $100 \%$ ) with lower values indicating higher self-control ability, thus lower falling probability. In the subsequent analysis, parameters are valued as following:

- Available corridor width 1 - $4 \mathrm{~m}$ (1 m, $1.5 \mathrm{~m}, 2 \mathrm{~m}, 3 \mathrm{~m}, 4 \mathrm{~m})$.

- Maximum falling probability at the edge (parameter $\alpha$ ) $30 \%-70 \%$ increased by $10 \%$ and with $15 \%$ standard deviation of the mean value

In Figure 3, the distribution of worker trajectory along a 1.0-meter width corridor is presented. The most frequent trajectory is in the middle of the corridor (half meter apart from the opening) with decreasing frequency towards the corridor edge. Similar distributions are obtained for other corridor widths and for $\alpha$ parameter. Table 1 illustrates the falling average probability for different values of width $\mathrm{x}$ and parameter $\alpha$. For example, for a corridor width $\mathrm{x}=1$ and $\alpha=25 \%-35 \%$, the fall average probability is $8.93 \%$. As expected, the fall probability is increased by the reduction of the corridor width and the increase of $\alpha$ and reaches the worst case at an approximately value of $21 \%$. These results may not be considered with their nominal values $(20 \%$ probability means one fall every five passes). Instead, the analysis is rather comparative and aims solely to differentiate among different scenarios. Nevertheless, the risk differs depending on operational conditions (e.g., load carriage) and a rather restrictive point of reference has been considered in this study. Besides the average probability, the falling probability distribution can also be developed in each case. Figure 4 shows the distribution for the 
case of $\mathrm{x}=1$ and $\alpha=25 \%-35 \%$. The distribution is not symmetrical having the most frequent value around $5 \%$ and the average value close to $9 \%$.

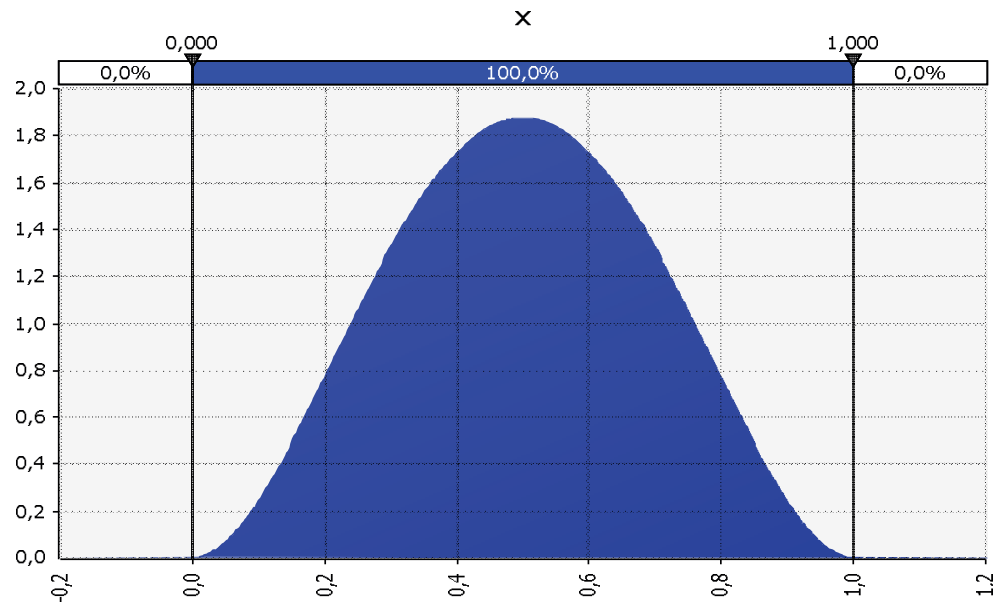

Figure 3: Probability distribution of worker trajectory in a $1.0 \mathrm{~m}$ width corridor

Table 1: Falling average probabilities for width $\mathrm{x}$ and parameter $\alpha$

\begin{tabular}{cccccc}
\hline & $\mathbf{x}=1.0 \mathrm{~m}$ & $\mathbf{x}=1.5 \mathrm{~m}$ & $\mathbf{x}=2.0 \mathrm{~m}$ & $\mathbf{x = 3 . 0} \mathrm{m}$ & $\mathbf{x}=4.0 \mathrm{~m}$ \\
\hline$\alpha: 25 \%-35 \%$ & $8.93 \%$ & $5.15 \%$ & $3.12 \%$ & $1.31 \%$ & $0.66 \%$ \\
$\alpha: 34 \%-46 \%$ & $11.96 \%$ & $6.88 \%$ & $4.16 \%$ & $1.76 \%$ & $0.88 \%$ \\
$\alpha: 42.5 \%-57.5 \%$ & $14.95 \%$ & $8.60 \%$ & $5.21 \%$ & $2.20 \%$ & $1.10 \%$ \\
$\alpha: 51 \%-69 \%$ & $17.89 \%$ & $10.32 \%$ & $6.22 \%$ & $2.65 \%$ & $1.32 \%$ \\
$\alpha: 60 \%-80 \%$ & $20.94 \%$ & $12.01 \%$ & $7.27 \%$ & $3.09 \%$ & $1.56 \%$ \\
\hline
\end{tabular}

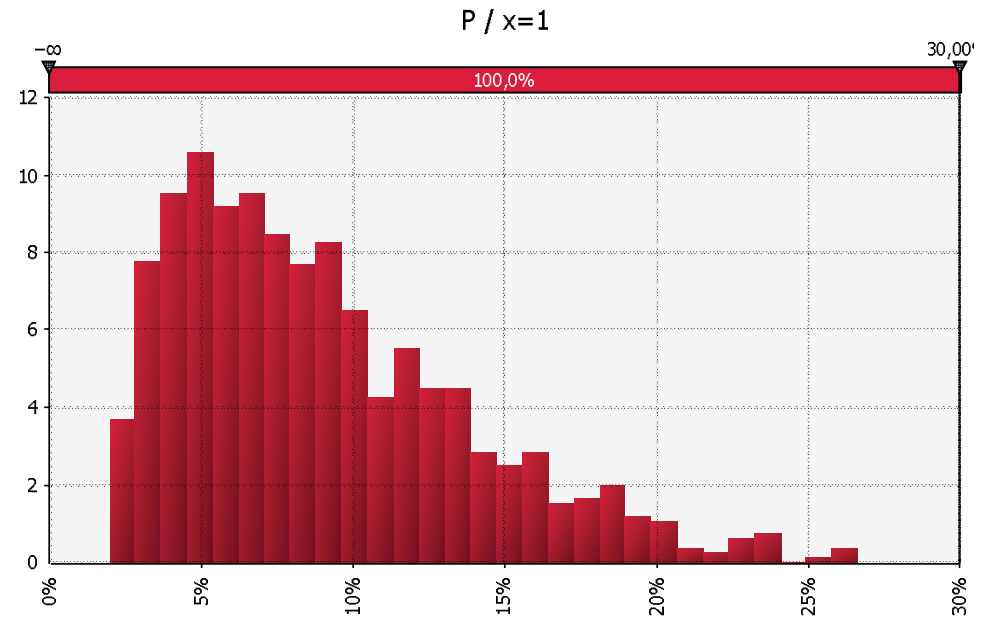

Figure 4: Falling probability distribution for width $\mathrm{x}=1 \mathrm{~m}$ and risk parameter $\alpha=0.30$

Table 2 illustrates the results associated with the three scenarios described in Figure 2. It can be observed that the risk probability increases as the effective corridor width is 
reduced (scenario 2) and as the fall risk parameter $\alpha$ is increased. Putting a barrier (or obstacle) along the opening drives the worker towards the safe side of the corridor and results in reduced risk probability. As expected, the existence of an object at the northwest corner of the opening (next to point D), preventing workers from approaching the opening, would be of highest importance as this is the "red" area for accident occurrence. Again the probability values presented in Table 2 should be considered as indicative rather than actual ones.

Table 2: Risk Probability under different scenario

\begin{tabular}{cccc}
\hline & Scenario 1 & Scenario 2 & Scenario 3 \\
\hline$\alpha: 25 \%-35 \%$ & $7,24 \%$ & $15,02 \%$ & $7,13 \%$ \\
$\alpha: 34 \%-46 \%$ & $9,66 \%$ & $20,04 \%$ & $9,52 \%$ \\
$\alpha: 42.5 \%-57.5 \%$ & $12,08 \%$ & $25,08 \%$ & $11,90 \%$ \\
$\alpha: 51 \%-69 \%$ & $14,49 \%$ & $29,99 \%$ & $14,28 \%$ \\
$\alpha: 60 \%-80 \%$ & $16,94 \%$ & $35,00 \%$ & $16,69 \%$ \\
\hline
\end{tabular}

Based on such analyses, the safety manager can evaluate alternative precaution measures to minimise the safety risk. Such measures can be the placement of a protective barrier or an object at the northwest corner of the opening, the stimulation to workers for one-way circular movement around the opening, or the use of an alternative route (southeast of the opening), if this is safer for the worker movement.

\section{CONCLUSIONS}

The aim of this work is the development of a tool for accident risk assessment in construction projects exploiting the capabilities of BIM in project and construction site representation and the mechanism of simulating the actual work conditions that may end up to accidents. The proposed method integrates BIM, Monte Carlo simulation, and a programming language for the development of the "virtual reality" scheme (visual C \#). Risk assessment outputs could then be linked to BIM to reflect high risk locations and periods. A basic interpretation (i.e. low, medium, high) can be used to create a BIM heat map of accident risk. Such a heat map, when also integrated with the schedule, could provide valuable insight on what is dangerous, when danger is posed, and where the danger resides. With this information, risk could be prioritized and preventively managed.

In this paper, the 'fall through an opening" risk is examined, however, other cases with machinery movement within the construction site have also been analysed. Although the proposed method presently focuses on certain type of risks, it provides the necessary insight and specifications to deal with other risks. These initial steps and experience can facilitate the expansion to a full-scale system. Once fully developed, the proposed system can be carefully field-tested to comprehend the implications that new feedback has on perception of safety risk, decision-making, collaboration, communication, and other safety-critical constructs to avoid undesired consequences. 


\section{REFERENCES}

Abraham, D.M., McGlothlin, J.D., Halpin, D.W., and Hinze, J. (2004). Construction safety alliance examining causes of construction injuries and defining best practices that improve safety performance. Constr. Inf. Quart. 6 (1), 9-16.

Carter, G. and Smith, S. (2006). Safety hazard identification on construction projects. Journal of Construction Engineering and Management, 132(7), 197-205.

Chi, S., Hampson, K., and Biggs, H. (2012). Using BIM for smarter and safer scaffolding and formwork construction: A preliminary methodology. Proceeding of the CIB W099 International Conference on "Modelling and Building Health and Safety", Singapore, 64-73.

Downey, J. (2012). Turner Construction Company's continuous improvement in jobsite safety: model based analysis and rule checking in Solibri for safety. Solibri Magazine 2012. <http://www.solibri.com/magazine/solibri-magazine-12012-published.html>

Goetsch, D. (2012). Construction Safety and Health, 2nd ed. Prentice Hall, USA.

Isaac, S., and Edrei, T. (2016). A statistical model for dynamic safety risk control on construction sites. Automation in Construction, 63, 66-78.

Kiviniemi, M., Sulankivi, K., Kahkonen, K., Makele, T., and Merivirta, M. L. (2011). BIMbased safety management and communication for building construction. VTT research notes, 2597.

$\mathrm{Ku}, \mathrm{K}$., and Mills, T., (2010). Research needs for building information modelling for construction safety. International Proceedings of Associated Schools of Construction 45nd Annual Conference, Boston, MA.

Rozenfeld, O., Sacks, R., Rosenfeld, Y., and Baum, H. (2010). Construction job safety analysis. Safety Science 48(4), 491-498.

Saurin, T.A., Formoso, C.T., and Guimaraes L.B.M. (2004). Safety and production: an integrated planning and control model, Construction Management and Economics 22 159-169.

Sulankivi, K., Mäkelä, T., and Kiviniemi, M. (2009). BIM-based site layout and safety planning. Proceedings of the First International Conference on Improving Construction and Use through Integrated Design Solutions, CIB IDS 2009, Espoo, Finland.

Sulankivi, K., Teizer, J., Kiviniemi, M., Eastman, C.M., Zhang, S., and Kim, K. (2012). Framework for integrating safety into Building Information Modelling. Proceedings of the CIB W099 International Conference on "Modelling and Building Health and Safety”, Singapore, 93-100.

Tam C.M., Tong T.K.L., Chiu, G.C.V., and Fung I.W.H. (2002). Non-structural fuzzy decision support system for evaluation of construction safety management system, International Journal of Project Management 20, 303-313.

Zhang, S., Teizer, J., Lee, J. K., Eastman, C. M., and Venugopal, M. (2013). Building information modeling (BIM) and safety: Automatic safety checking of construction models and schedules. Automation in Construction 29, 183-195.

Zhang, S., Sulankini, K., Kiviniemi, M., Romo, I., Eastman, C., and Teizer, J. (2015). BIMbased fall hazard identification and prevention in construction safety planning. Safety Science 72, 31-45. 